\title{
STUDIES ON PAIN : AN INVESTIGATION OF SOME QUANTITATIVE ASPECTS OF THE DOL SCALE OF PAIN INTENSITY
}

\author{
By JAMES D. HARDY, HAROLD G. WOLFF, AND HELEN GOODELL \\ (From the Russell Sage Institute of Pathology, The New York Hospital, and the Departments \\ of Physiology, Medicine (Neurology) and Psychiatry, Cornell \\ University Medical College, New York City)
}

(Received for publication December 22, 1947)

Wolff and his collaborators (1) have, for a number of years, reported estimates of intensity of pain in terms of one to ten "plus," one plus representing a minimal pain and ten plus the worst pain ever experienced by the subject. This method was valuable as a means of roughly estimating relative pain intensities, especially in studies of experimental headache. Others have ressorted to procedures such as squeezing the biceps muscis or placing pressure on the styloid process (2), to produce pain of high intensity which the patient was asked to compare with his spontaneously occurring pain. Although the estimates of pain intensity based upon these procedures have been useful, the stimuli have lacked measurable aspects, and, therefore, have not been suitable for a quantitative study of pain intensity. In general, methods of estimating the intensity of painful sensations have suffered from two principal defects. First, it has been difficult for the investigator to separate from the entire pain experience the property of a painful sensation which is intensity. Indeed, at least one thoroughgoing investigator was led to the conclusion that the difficulties arising in this respect did not permit reliable estimates of pain intensity to be made (3). Second, the study of pain has been inadequate as regards the intensity aspect of the sensation, and no common basis has been provided for intercomparison of experimental data obtained under various circumstances in different laboratories.

In a previous communication (4) a scale of pain intensity was proposed, based upon the ability of normal individuals to discriminate differences in intensity of painful sensations. The unit of this scale was chosen arbitrarily as the sum of two just noticeable differences, and the term "dol" was suggested as the name of the unit of pain intensity. Three important questions concerning the dol scale are: First, does the sum of the two just noticeable differences represent the same difference in pain intensity in all parts of the dol scale? Second, are the numbers representing the pain intensity in dols associative, that is, is a four-dol pain twice as intense as a two-dol pain, etc.? And third, is the scale sufficiently representative of common experience to serve as a basis for intercomparison of data? Reported herein is evidence indicating that the above questions should be answered affirmatively.

The dol scale was first established when, using a three-second exposure of radiant heat on the skin as the stimulus, the difference in intensity of radiation which elicited a just noticeable difference in pain sensation (jnd) was measured. Measurements were begun with the pain threshold stimulus $\left(220 \mathrm{mc} / \mathrm{sec} / \mathrm{cm}^{2}\right)$ and carried to progressively greater stimulus intensities. As the intensity of radiant heat was increased beyond that causing tissue damage, greater increments of stimulus were required to elicit distinguishable differences in pain intensity. For example, the pain elicited by a stimulus of $480 \mathrm{mc} / \mathrm{sec} / \mathrm{cm}^{2}$ could barely be differentiated from that elicited by 680 $\mathrm{mc} / \mathrm{sec} / \mathrm{cm}^{2}$, and greater amounts of stimulus elicited no distinguishable increase in pain intensity. Between the threshold stimulus and the maximum stimulus which could be discriminated, there were found to be 21 just noticeable differences. The dol was defined as the difference in pain sensation evoked by stimuli differing in intensity by the sum of two jnd's. The 21 jnd's between the threshold sensation and the "ceiling" pain were thus equivalent to ten and onehalf dols. It should be emphasized that painfulness, measured in dols, refers only to the intensity aspect of pain sensation and is estimated from the intensity of the stimulus which is the directly measurable quantity (millicalories per second per square centimeter).

It was recognized that the number of millicalories per second per square centimeter required 
to produce a one-dol change in pain intensity increased with the intensity of the stimulus. However, it was hoped that the size of the dol would be the same in terms of painfulness throughout the scale, in as much as it might be logically assumed that a just noticeable difference in painfulness would be the same for a mild pain as for a severe pain. Indeed, this was Fechner's argument when, in the last century, he made his wellknown modification of the Weber Law (5). For example, recent studies of hearing (6) show that for loudness, the intensity attribute of hearing, the sum of the jnd's does not constitute a uniform sensory scale. That is, the scale of loudness based on the sum of jnd's does not coincide with the scale derived from estimating the relative intensity of sounds in terms of a sound of fixed intensity. It is, therefore, evident that in the case of loudness at least, Fechner's assumption that $a$ jnd is of the same sensory magnitude regardless of the intensity of sensation is not valid, and that the jnd for loudness is smaller for low intensities of sound and larger for the high intensities of sound.

The technique of comparing the intensity of one sensation with another has been termed by the psychologists "fractionation" and this consists of requiring the subject to estimate the intensity of a sensation in terms of fractions or multiples of a standard, fixed intensity. It was desirable, therefore, to investigate pain by means of the fractionation methods and to ascertain whether or not the size of the dol was uniform throughout the scale of painfulness.

It is the purpose of this communication to report the results of studies in the estimation of painfulness by means of fractionation, and to compare this method of estimating pain intensity with the scale of pain set up on the basis of the jnd's.

\section{METHOD}

The painful sensation studied in these experiments was produced in the skin by a three-second exposure to an intense thermal radiation. The apparatus used to heat the skin has been previously described (4) and mention is made here only of the necessity of checking the instrument's calibration at frequent intervals with a standardized radiometer. Our practice has been to calibrate the instrument before each experiment, and data so obtained show that, as the heat lamp ages, its radiant heat output decreases for any given reading of the voltmeter.
This effect may possibly be due to the gradually increasing opacity of the glass bulb as the result of distillation of metal from the incandescent filament.

In one series of experiments the authors served as both experimental subjects and observers; and in a second series 70 medical students conducted carefully supervised experiments on each other, acting in turn as subject and observer.

Fractionation of the painful sensation was accomplished in the following manner. Each subject was exposed at the beginning of an experiment to a standard stimulus evoking a pain which on the scale of just noticeable differences corresponded to eight dols. Subjects were then exposed to eight intensities of stimulus chosen to evoke pain intensities of $1,2,3, \ldots 8$ dols. Three stimuli at each of the eight intensity levels were given in random order. The subjects were asked to report the intensity of the pains in terms of fractions of the initial, standard pain. As a rule, the standard stimulus was repeated once during the course of the experiment to refresh the subject's memory. Care was taken not to overstimulate an area of the skin, as the skin becomes hypersensitive as the result of too rapid stimulation. An interval of at least five minutes is desirable between stimuli.

\section{RESULTS}

The pain intensities reported in Series 1 are shown in Figure 1. This represents 72 tests on three subjects. The scatter of the reports is about one dol on either side of the average report for a particular stimulus intensity, and reports as much as one and one-half or two dols from this average were not uncommon. As the experiments proceeded it became apparent that the experience

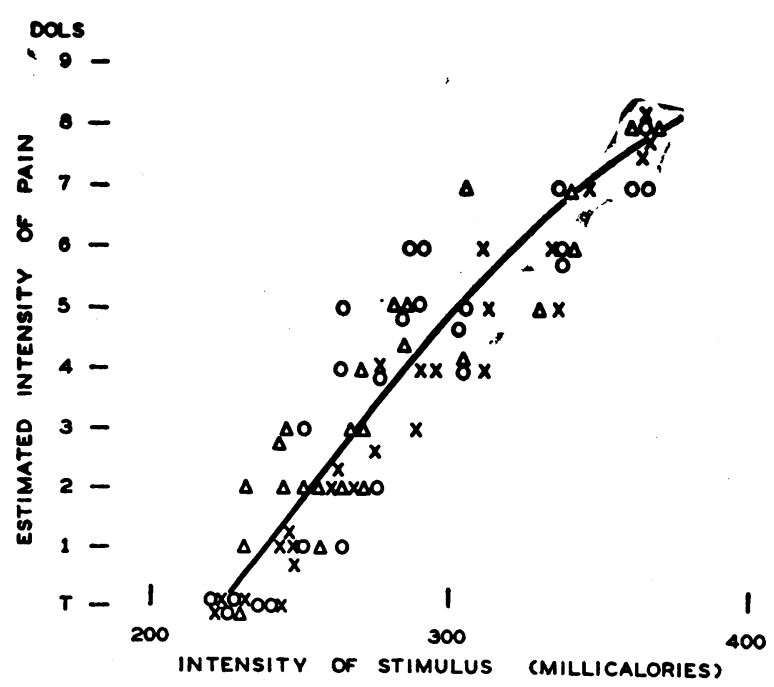

Fig. 1. Estimates of Pain Intensity as Fractions of a Pain of Eight Dols Intensity 


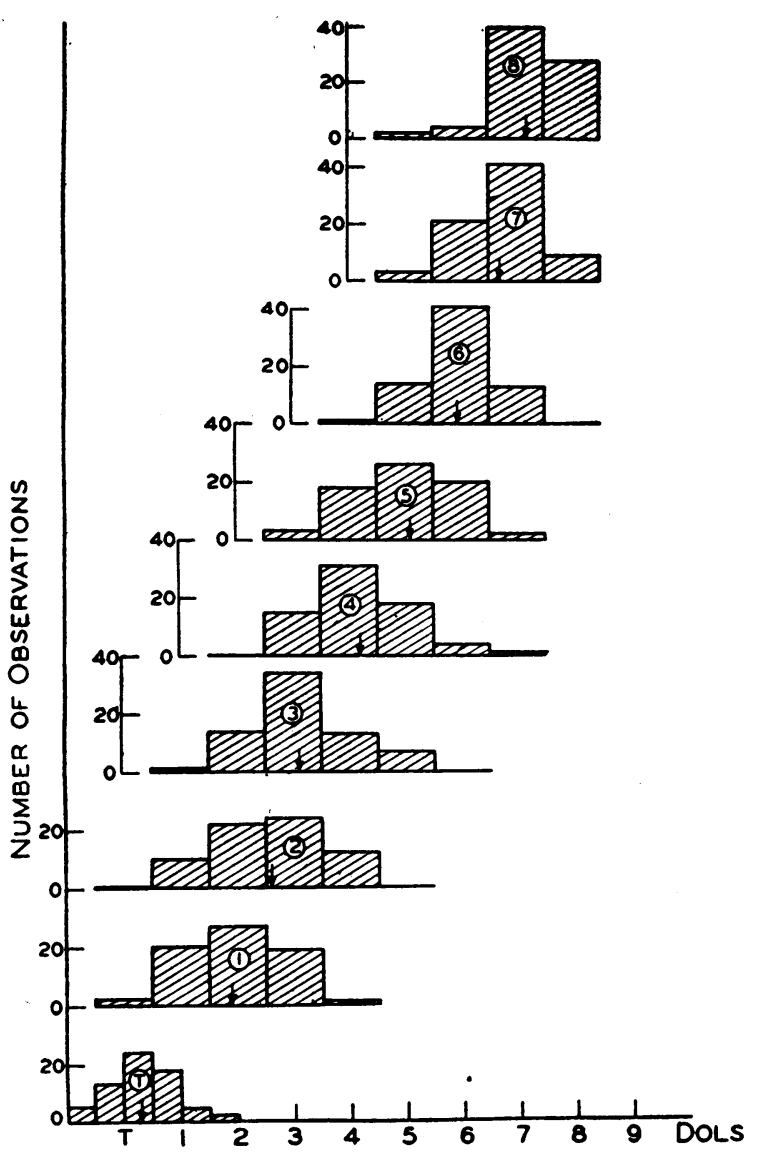

Fig 2. Distribution Diagrams of Reports of 70 Medical Students Reporting Pain Intensities as Fractions of Eight Dols

of the subjects, as indicated by variations in reports, did not increase the accuracy of reporting. Lack of concentration upon the procedure was the most important single factor causing variability in reports. Memory was not a significant factor because the accuracy, of reporting showed no change when in the course of the experiment the standard was presented repeatedly. The heavy line in the figure represents the dol scale based on measurements of the just noticeable differences, and this line coincides exactly with the average of the reports for all the stimuli given (see also Figure 4).

The second group of experiments, done by the medical students under supervision; indicates the scatter of reports from untrained but intelligent subjects and observers. The students followed the procedure outlined above and the results are shown in Figure 2 as a series of scattergrams. In general, the average of the reports of the intensity of pain evoked by each unknown stimulus was within one dol of the value determined by the method of just noticeable differences and the scatter of the reports was approximately the same as that obtained with experienced observers.

These results may be compared with those of experiments done in this laboratory prior to the evolution of a scale of painfulness, because, in the course of several years' experimentation with the thermal stimulus for producing pain, reports were made from time to time on the intensity of pain elicited by various intensities of thermal radiation. In these reports as well as in those for estimating headache (2), intestinal pain (7), pain from immersion of an extremity in cold water (8), etc., it has been the practice for subjects to make reports of pain intensity in "pluses," using an arbitrary scale of one to ten plus to represent the intensities of pain between threshold and the most intense pain experienced. This procedure is essentially fractionation based on the individual's life experience. The results of 45 such estimates of pain intensity made over a period of several months by three subjects are shown in Figure 3.

It will be noticed that although ten plus was considered to be the most intense pain ever exexperienced, reports of $11+$ and $12+$ were made

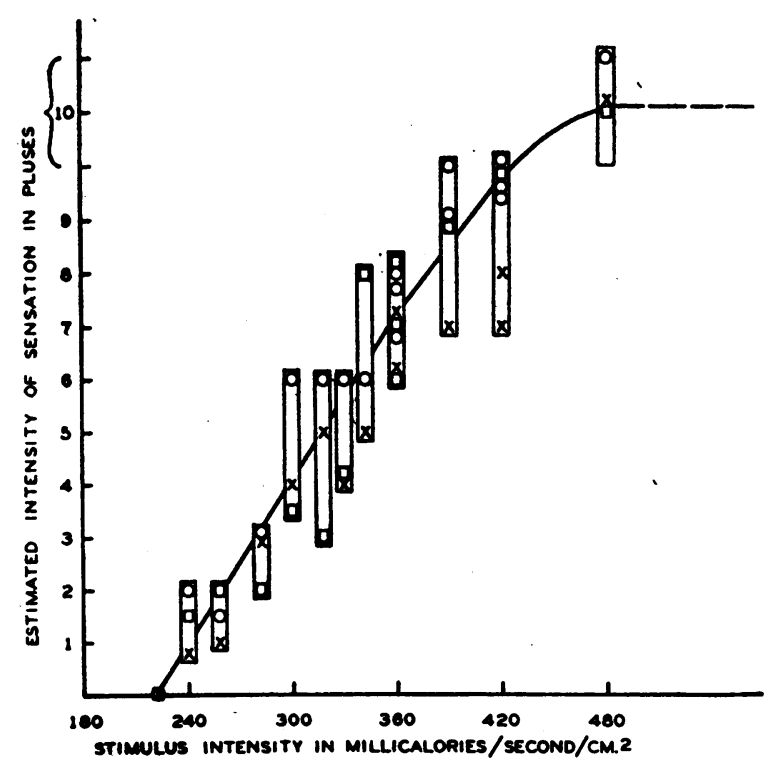

Fig. 3. Estimates of Pain Intensity in Terms of the Most Intense Pain Ever Experienced 


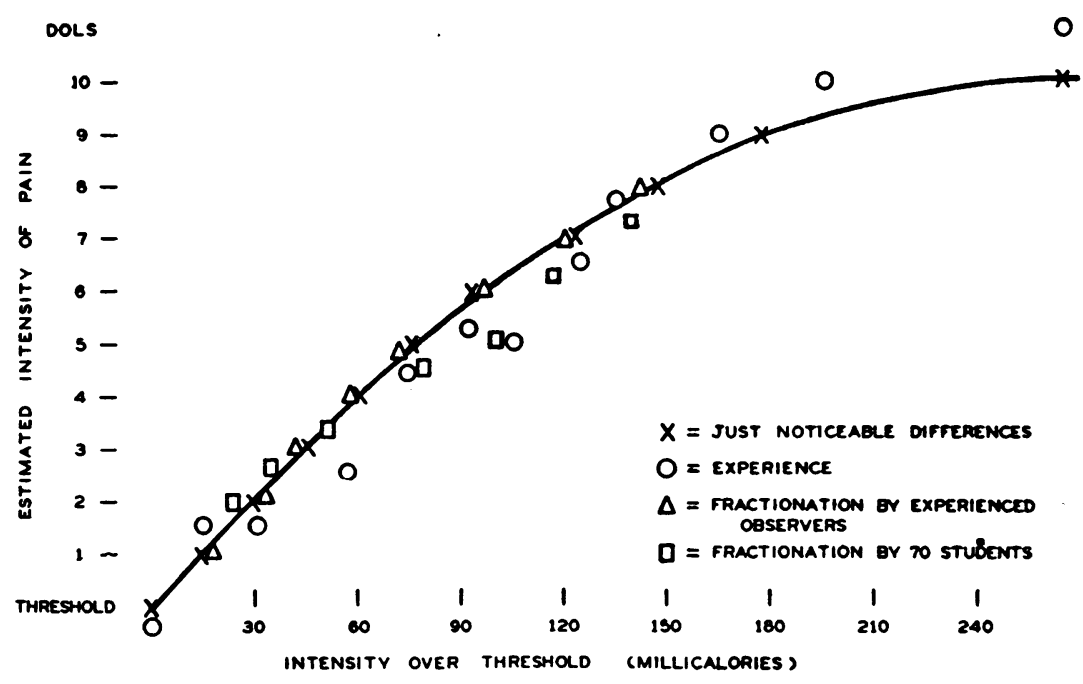

Fig. 4. A Comparison of Methods of Estimating Painfulness

(a) (X) Integration of the just noticeable differences. (b) ( $\square, \Delta$ ) Fractionation of an eight-dol pain by 70 medical students and three experienced observers. (c) (๑) Fractionation of the most intense pain experienced.

for the most intense stimuli. This was due to the lack, at that time, of any concrete standard pain with which to compare the painfulness of these intense stimuli, and the subjects were consciously "stretching" the imaginary scale as it was evident to them that they could experience more degrees of painfulness than they had imagined. The solid line drawn in Figure 3 again refers to the scale of pain previously suggested from studies of the just noticeable differences, equating one "dol" to one "plus" value. The agreement between the dol scale and the "plus" values is close except at the highest intensities.

A summary of the various methods of estimating the intensity of the experimental pain is shown in Figure 4. The heavy line is drawn through the points determined by adding the jnd's. The results obtained with the three experienced observers coincide with this line and the observations made by the students are near the line although with more deviation. The judgments from the plus scale are included to illustrate the fact that these judgments based on the life experience of the subject are in close agreement with the dol scale of pain.

A third series of experiments was designed to ascertain the effect of fatigue, minor mood changes and feelings of general effectiveness upon the perception of pain. It had been previously demon- strated that these variables did not affect pain threshold measurements (9). Three subjects were studied almost daily over a period of six weeks in the following manner. At about noon each day the subjects reported to the laboratory for testing. A record was made of the mood, feeling of effectiveness, and of any unusual activity or experience in the subject's life during the past 24 hours. Following this record, pain thresholds were measured and three unknown stimuli were given. A standard stimulus was not presented for comparative purposes in these experiments. The subject made a report of the intensity of the pain evoked. The unknown stimuli were distributed at random between the threshold stimulus and that causing a nine-dol pain. The ten-dol stimulus was avoided because it produces tissue damage.

The results of this study are shown in Figure 5 in terms of deviations from the report expected on the basis of the dol scale. The deviations are plotted as positive or negative according to whether the reports were greater or less than the dol scale values. Each point is the average of the three reports of the subject for the day in question. The average deviation of the reports from the dol scale is minus one-half dol, that is, the subjects underestimated rather than overestimated the intensity of the stimuli. The maximum deviation of 


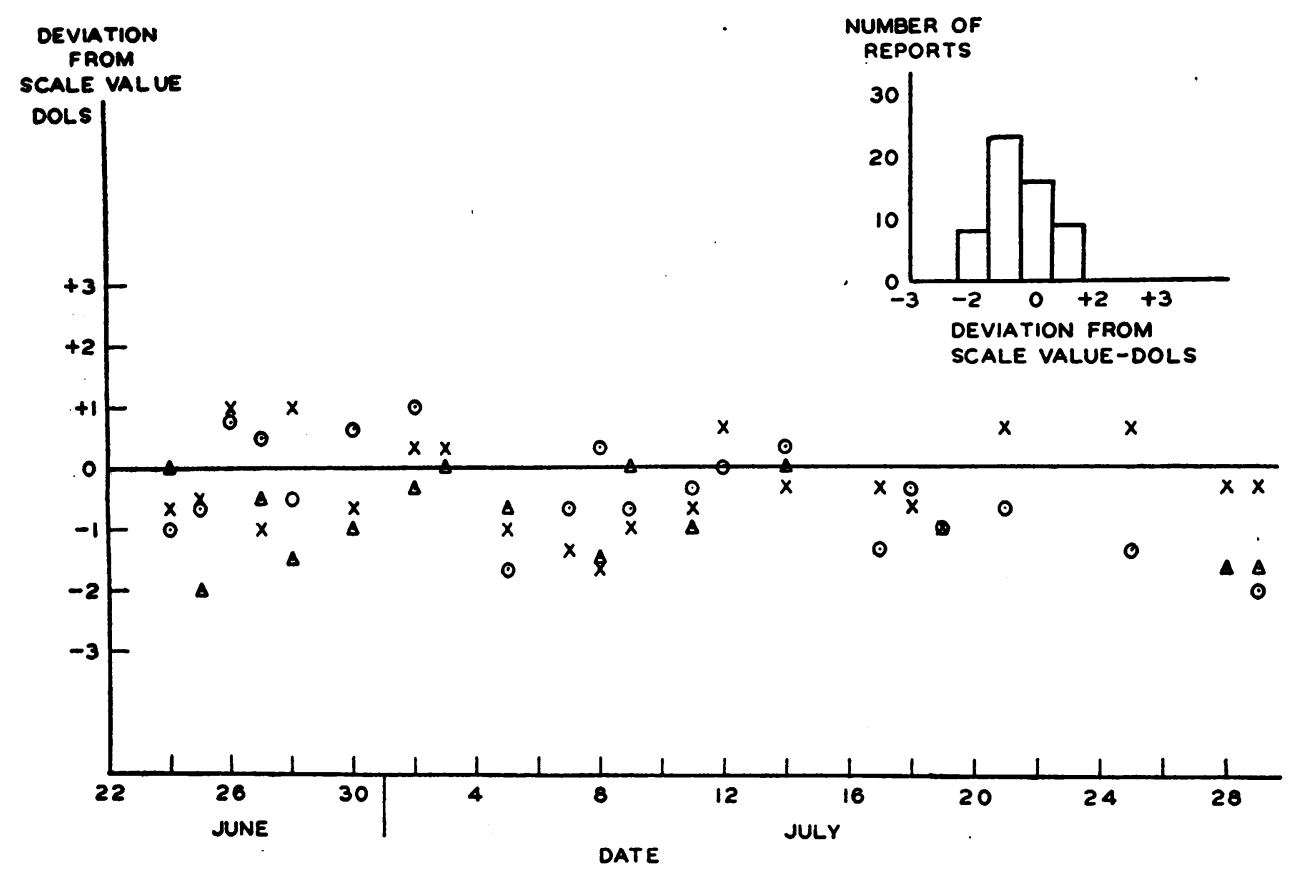

Fig. 5. Day to Day Variations in Reporting Pain Intensity by Three Subjects

the reports from the scale value was two dols and the mean deviation plus-minus one-half dol. Estimates of mood and of general effectiveness in the three subjects varied from day to day. Fatigue resulting from 30 hours of wakefulness was encountered in one subject, and severe disappointment in another. Tenseness, mild depression, exhileration, contentment were reported on different days. These mood changes and fatigue did not appear to be associated with any predictable change in the ability of the subject to estimate pain intensity. This result agrees with the observations previously made on the pain threshold (9).

\section{DISCUSSION}

The data presented in Figures 1 and 2 indicate that there is a variation of plus-minus 10 per cent in a single measurement of pain intensity obtained by this method. The accuracy of estimation does not depend upon the subject's experience with the method and it is possible that pressing a subject too hard for accurate reports will lead to a greater scatter in the results than is otherwise obtained.

Without a standard pain for comparison pain intensities at high levels are difficult to estimate, as shown in Figure 3. Furthermore, it is important to call the subject's attention to the fact that it is the intensity aspect of the sensation which is being estimated. The overall distress of the individual, in which the uncertainty as to duration of the pain, the amount of tissue involved and the implications of the situation are of major importance, should not be confused with intensity of pain.

It is shown in Figure 4 that the scales of painfulness, as determined by fractionation of intensity, and by integration of the just noticeable differences, coincide. This fact implies that for cutaneous pain sense, the sensory magnitude of jnd's is the same in all parts of the dol scale. That is, increasing the painfulness by a jnd at one dol causes the same change in sensation as increasing the painfulness by the same amount at eight dols. For example, although this type of relationship does not hold for the loudness of sound, it does hold true for the perception of the pitch of sound, keeping the loudness constant. The fact that the pain scales coincide would a priori make it seem likely that judgments of pain intensity, whether made on the basis of increments of increasing intensity or in fractions of a "top" pain, would lead to the same results. It is, therefore, not surprising that estimates of pain intensity in terms of "pluses" as used by Wolff and his associates agree in relative value with the dol scale, in 
spite of individual differences in the methods by which the observers may have arrived at their estimations of pain intensity. The numbers of the pain scale which represent the intensity aspect of pain are capable of being added and divided according to the ordinary rules of arithmetic, and it is possible to define one dol both as the sum of two jnd's and as one-tenth of the intensity of the ceiling pain. It is proper, also, to speak of one pain as being either "twice as strong" as another or as equivalent in intensity to the sum of two smaller pains. This suggests that there is only one scale of pain intensity and that the estimates by normal subjects on the basis of a standard pain will result in reports which can always be evaluated in terms of the dol scale.

The agreement of the estimations of pain intensity from general experience with the more carefully determined dol scale is of significance. It lends validity to the use of such a scale of "pluses" as a quantitative procedure in past experiments on headache, etc. Also, it implies that the clinician's interpretation of the patient's estimates of pain in terms of "mild," "moderate," "severe" and "extreme" represents fairly reproducible divisions of the intensity scale of pain. Thus, a "mild" pain is probably one that has an intensity between threshold and two dols, a pain such as is usually abolished by the use of acetylsalicylic acid or some other coal tar derivative. A "moderate" pain is one of intensity between three and six dols, and is a pain that cannot be tolerated for any length of time without loss of sleep and disturbing reactions. Codeine and other opiates are usually required to manage a pain of this intensity. A "severe" pain has an intensity between six and nine dols and maximal amounts of the most effective analgesics are required to reduce such pain to a threshold level (10). An "extreme" pain, nine to ten and one-half dols, will require complete anesthesia, either general or locally applied to the source of pain, to bring relief.

A further point of interest in this connection is the accuracy with which judgments can be made of the intensity of a pain from memory of one's general experience. It is as if a normal adult had in his mind at all times a keen sense of the intensity aspect of pain, so that a person without experience in making judgments and discriminations of this sort can give a surprisingly accurate estimate of the intensity of a pain. Pain memory is apparently very good, and is undoubtedly important to the body economy.

Over-reaction to pain, due to anxiety and fixed attitudes toward pain sensation was encountered in four of the 70 medical students. These at first exhibited an almost complete lack of discriminatory ability during the observations, often reporting a one-dol pain as six dols and vice versa. Also, pain threshold measurements were difficult to make on these individuals. It was apparent that the attitude of these subjects toward the experiment and their fellow student observers prevented their making proper estimations. These four subjects were studied individually by the authors following their class experiments, and, after several trials, normal reports were usually obtained. Two of these four subjects had suffered major physical injuries and had experienced pain over long periods of time. Similar difficulties in estimating pain intensities, and in recognizing the pain threshold, were sometimes encountered in patients. However, with reassurance and repeated trials, reproducible results were obtained.

\section{SUMMARY AND CONCLUSIONS}

1. Using a three-second exposure of thermal radiation on the skin as a painful stimulus, measurements have been made of the stimulus intensities which evoked painful sensations of various relative magnitudes. Three series of experiments were performed, in the first of which three experienced observers reported the relative intensities of pain in terms of fractions of an eight-dol pain. In the second series of experiments 70 medical students were similarly studied. In a third series of experiments the effects of fatigue and minor mood changes upon discriminations of relative intensity of pain were studied.

2. It has been ascertained that the scale of painfulness based on estimations of pain intensity as fractions of a known pain, coincides with the scale based on the summing of just noticeable differences in pain sensation. The dol as a unit of painfulness can thus be defined as the sum of two jnd's in pain sensation, or as approximately onetenth the intensity of the ceiling pain.

3. The accuracy of estimating pain intensity is limited by the ability of the individual to discrimi- 
nate differences in pain intensity. This limit is plus-minus one-half dol; the spread of reports about a mean value was approximately plus-minus one dol. Experience in reporting pain intensities did not increase the accuracy of estimation.

4. Moderate fatigue and day to day variations in mood were not associated with an appreciable change in the ability to estimate pain intensity in three normal subjects tested over a period of six weeks.

5. From the above studies it is concluded that the dol scale provides a satisfactory basis for estimating pain intensity in the following respects:

a. It provides a numerical scale of sensory steps all of which are equal, even though the stimuli differences corresponding to these steps are not equal.

b. It is based on a type of stimulus which gives reproducible results in terms of threshold measurements.

c. It affords a basis for the intercomparison of other methods of estimating pain intensity, providing these estimates have been made in terms of a reproducible pain.

\section{BIBLIOGRAPHY}

1. Clark, D., Hough, H. B., and Wolff, H. G., Experimental studies on headache: observations on head- ache produced by histamine. Arch. Neurol. \& Psychiat., 1936, 35, 1054.

2. Libman, E., Observations on sensitiveness to pain. Tr. Assn. Am. Phys., 1926, 41, 305.

3. Lewis, T., Pain. The Macmillan Co., New York, 1942.

4. Hardy, J. D., Wolff, H. G., and Goodell, H., Studies on pain: discrimination of differences in intensity of a pain stimulus as a basis of a scale of pain intensity. J. Clin. Invest., 1947, 26, 1152.

5. Culler, E. A., Thermal discrimination and Weber's Law. Arch. Psychol., 1926, 13, 81.

6. Stevens, S. S., and Davis, A. H., Hearing: Its Psychology and Physiology. John Wiley \& Sons, New York, 1938, p. 110.

7. Wolf, S., and Wolff, H. G., Human Gastric Function: an experimental study of a man and his stomach. Oxford University Press, New York, 1943 and 1947.

8. Wolf, S., and Hardy, J. D., Studies on pain : observations on pain due to local cooling and on factors involved in the "cold pressor" effect. J. Clin. Invest., 1941, 20, 521.

9. Schumacher, G. A., Goodell, H., Hardy, J. D., and Wolf, H. G., Uniformity of the pain threshold in man. Science, 1940, 92, 110.

10. Wolff, H. G., Hardy, J. D., and Goodell, H., Studies on pain: measurement of the effect of morphine, codeine and other opiates on the pain threshold and an analysis of their relation to the pain experience. . J. Clin. Invest., 1940, 19, 659. 